\title{
Development of severe hyponatremia due to cerebrospinal fluid leakage following meningomyelocele surgery in a newborn
}

\author{
Ismail Kürşad Gökce, MD, ${ }^{1}$ Hatice Turgut, MD, ${ }^{1}$ Ramazan Ozdemir, MD, ${ }^{1}$ and \\ Selami Cagatay Onal, MD²
}

1Department of Pediatrics, Division of Neonatology, and 2Department of Neurosurgery, Inonu University School of Medicine, Turgut Ozal Medical Center, Battalgazi, Malatya, Turkey

\begin{abstract}
Cerebrospinal fluid leakage following meningomyelocele surgery is a frequent complication in the wound-healing period and is associated with wound dehiscence. CSF loss can cause severe hyponatremia, especially in the newborn and early infancy periods when dietary sodium content is relatively low. Hyponatremia in the newborn period can result in adverse neurodevelopmental outcomes. In addition, hyponatremia's cerebral effects can increase complications in neurosurgery patients. The authors present the case of a newborn in whom CSF leakage from the operative site and severe hyponatremia developed following meningomyelocele surgery. To the best of their knowledge, severe hyponatremia caused by CSF leakage after meningomyelocele surgery has not been previously reported in the literature.
\end{abstract}

https://thejns.org/doi/abs/10.3171/2017.11.PEDS17386

KEY WORDS hyponatremia; meningomyelocele; newborn

$\mathrm{W}$ HEN a baby with a meningomyelocele is born, the ideal approach is to close the meningomyelocele sac and treat accompanying pathologies, if any, within the first 24 hours. ${ }^{4,7}$ Early and late complications after meningomyelocele surgery are closely associated with wound dehiscence. Cerebrospinal fluid leakage is a common complication following meningomyelocele surgery during the wound-healing period. ${ }^{4}$ Hyponatremia related to CSF leakage has been reported to occur in preterm infants following external ventricular drainage to treat posthemorrhagic hydrocephalus. ${ }^{8}$ Hyponatremia can lead to adverse neurodevelopmental outcomes in the newborn period..$^{11}$ It is a serious complication, especially in neurosurgery patients because it can give rise to additional problems such as cerebral edema, altered mental status, and vasospasms. ${ }^{13}$ Herein, we describe the case of a newborn child with absence of suckling behavior who was admitted on the 14th day (postnatal day 15) after meningomyelocele surgery with a CSF leak and severe hyponatremia (serum sodium level $104 \mathrm{mEq} / \mathrm{L}$ ).

\section{Case Report}

A 15-day-old girl was admitted to the emergency de- partment with drowsiness and decreased suckling for 3 days. The meningomyelocele surgical incision site had opened 3 days earlier, and the girl's suckling behavior had decreased gradually thereafter. The drowsiness had begun 2 days before admittance. According to the prenatal history, the infant was born to a 28-year-old mother (fourth pregnancy) via the normal vaginal route in the 40th gestational week. The infant had a birth weight of $2830 \mathrm{~g}$, and her parents had third-degree consanguinity. All the other siblings were healthy.

The patient had undergone surgery for a lumbar meningomyelocele in our hospital on the 1st postnatal day. According to the hospital records, her head circumference was $34 \mathrm{~cm}$ (25th-50th percentile). She had a full blood count preoperatively, and the results of serum electrolyte, liver, and kidney function tests were all within the normal ranges. The lateral ventricles were shown to be slightly dilated in the occipital area on a preoperative cerebral CT scan and on a CT scan obtained on postoperative day 3 . Millimeter-sized dense hemorrhage foci were seen in the 3 ventricle walls (Fig. 1A and B). According to the operative records, duraplasty was performed by bilaterally dissecting the dorsolumbar fascia after excising a $6 \times 6-$ $\mathrm{cm}$ meningomyelocele, and the skin folds were primarily 

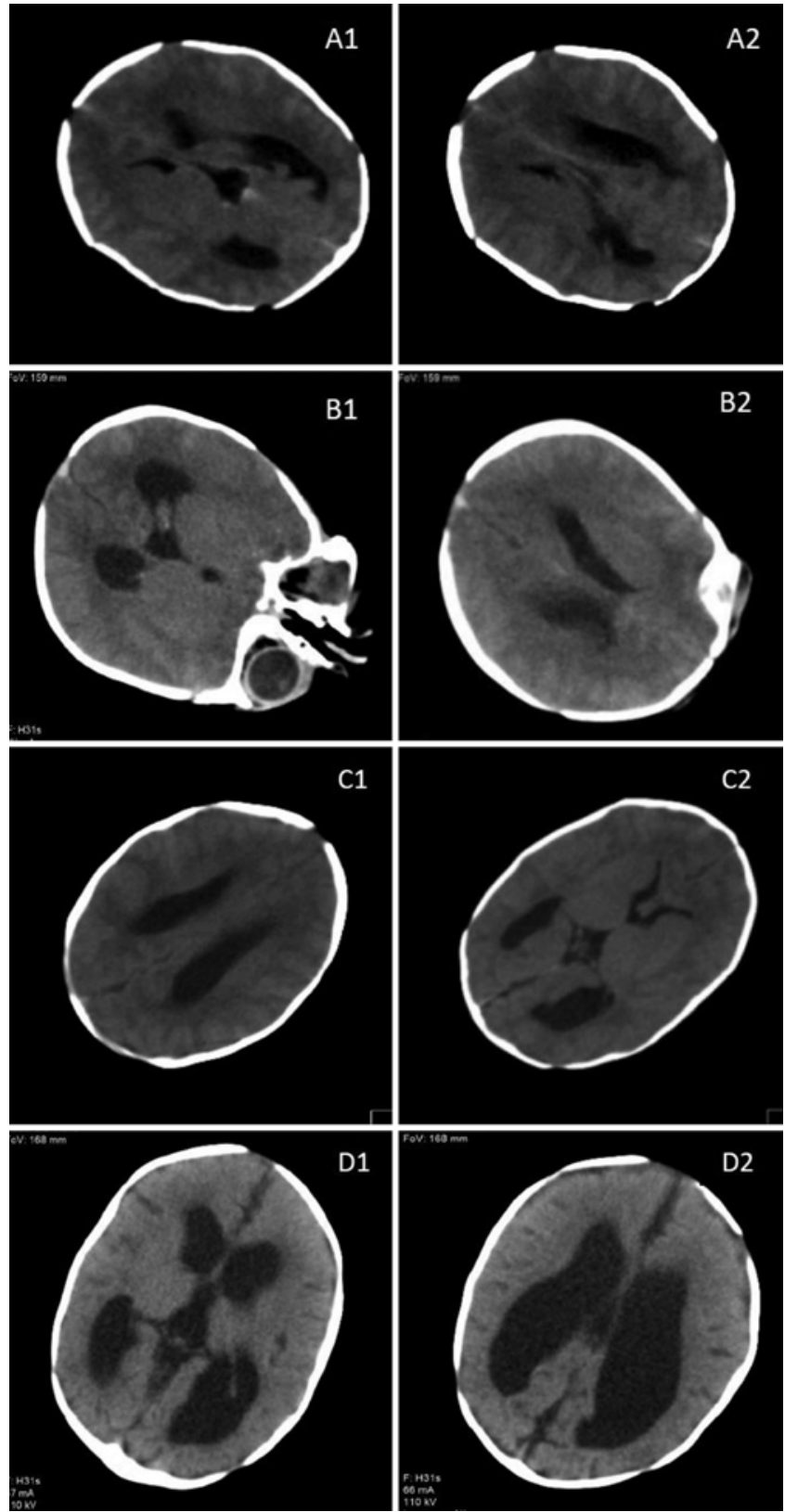

FIG. 1. Axial CT scans obtained preoperatively (A), 3 days postoperatively (B), 1 week after readmission (C), and 58 days after birth (D).

sutured. The patient had been discharged on the 7th postnatal day with a clean wound site and was on full enteral feeding. She was scheduled to attend an outpatient visit in the clinic 3 days after discharge.

Physical examination revealed the following: body weight of $2300 \mathrm{~g}$ (below 3rd percentile), head circumference of $34.5 \mathrm{~cm}$ (25th-50th percentile), body temperature of $36.2^{\circ} \mathrm{C}$, pulse of 136 beats/minute, respiratory rate of 50 breaths/minute, and blood pressure of $56 / 35 \mathrm{~mm} \mathrm{Hg}$. The patient had a cachectic, hypotonic, and lethargic appearance. A break of $4 \times 5 \mathrm{~cm}$ in the incisional site was observed, in addition to a yellow, aqueous discharge (Fig. 2 upper). The anterior fontanelle measured $2 \times 2 \mathrm{~cm}$, with
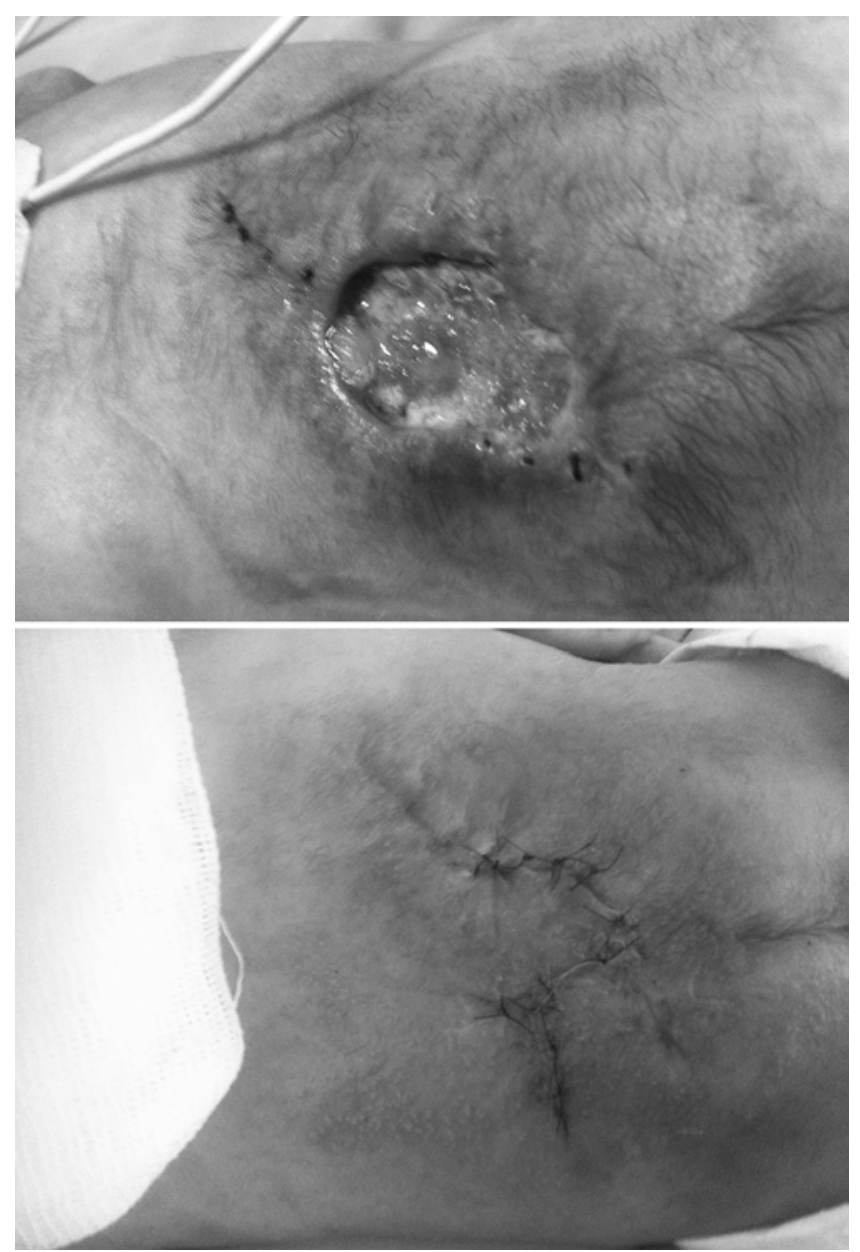

FIG. 2. Photographs showing a break in the incision site on the 15 th postnatal day (upper) and the skin defect closed on the 52nd postnatal day (lower).

normal bulging. Tonus and movements of the lower extremities were decreased. The findings of all other system examinations were normal.

Laboratory investigations revealed severe hyponatremia (serum sodium level $104 \mathrm{mEq} / \mathrm{L}$ ) and hypochloremia (serum chlorine level $71 \mathrm{mEq} / \mathrm{L}$ ). The other laboratory findings were as follows: potassium $6.1 \mathrm{mEq} / \mathrm{L}$; blood urea nitrogen $53 \mathrm{mg} / \mathrm{dl}$; creatinine $0.7 \mathrm{mg} / \mathrm{dl}$; and uric acid $11 \mathrm{mg} / \mathrm{dl}$. A complete blood count and C-reactive protein values were normal. In the urinalysis, the urine density was 1006, and urine sodium, which was measured twice, was $<20 \mathrm{mmol} / \mathrm{L}$. Serum levels of cortisol and ACTH were normal, and findings on renal and cranial ultrasound examination were normal.

The patient was given $140 \mathrm{ml} / \mathrm{kg} /$ day of fluid based on her birth weight and 12-hour sodium deficit therapy to increase the sodium value by $10 \mathrm{mEq} / \mathrm{L}$. After collecting blood and wound cultures, treatment was initiated with cefotaxime and vancomycin. After 12 hours, the patient's sodium level was $113 \mathrm{mEq} / \mathrm{L}$. Over the next 24 hours, sodium-deficit therapy was administered again to raise the sodium level by $10 \mathrm{mmol} / \mathrm{L}$. The control sodium value 
was $127 \mathrm{mEq} / \mathrm{L}$. Escherichia coli was isolated from the wound culture. Based on the patient's antibiogram, cefotaxime was stopped, and the patient received meropenem. Over the following days, the CSF leak continued at a rate of approximately 100-120 ml/day; this was measured by weighing the sterile pads on which we closed the lesion 3 times a day. Hydrocephalus was not observed on CT scans (Fig. 1C), although the CSF leakage continued. The patient received full enteral feeding. Additional sodium support (20-25 mEq/day) was provided, with $3 \%$ sodium chloride solution added to the enteral feeding solution. Wound dressing continued with rifampicin and nitrofurazona pomada. Vancomycin therapy was continued for 10 days, and meropenem was given for 14 days. Subsequently, prophylactic cefotaxime was initiated. At the end of this period (on postnatal day 33), the patient's CSF leakage was significantly reduced. Serial ultrasound measurements obtained during this period revealed no findings indicative of hydrocephalus, such as an abnormal increase in head circumference. The patient was followed up by the plastic surgery and neurosurgery departments. The skin defect was closed on postnatal day 52 (Fig. 2 lower). A small CSF leak from the suture site continued after the operation, and the patient developed hydrocephalus on day 58 (Fig. 1D). A ventriculoperitoneal shunt was placed. Sodium support was discontinued, and the CSF leak resolved after the shunt insertion. Serum sodium levels remained stable, and the patient was discharged.

\section{Discussion}

Hyponatremia is defined as a serum sodium level below $133-135 \mathrm{mmol} / \mathrm{L}$. It has a reported incidence of $25 \%-$ $65 \%$ in newborns admitted to the intensive care unit. ${ }^{9,13}$ Hyponatremia accompanying primary disease is associated with adverse outcomes in all age groups. It is seen in about half of neurosurgery patients, and the cerebral effects of hyponatremia can increase the rate of complications such as convulsions, cerebral edema, altered mental status, and vasospasms. ${ }^{2,13}$

The 2 most common causes of hyponatremia in neurosurgery patients (especially those with traumatic brain injury or subarachnoid hemorrhage, as well as those who have undergone transsphenoidal pituitary surgery) are inappropriate antidiuretic hormone secretion and cerebral salt loss, in contrast to hyponatremia associated with CSF loss, which has rarely been reported. After meningomyelocele surgery, CSF leakage and wound site infections are a common complication during the healing period, with a general incidence of $2 \%-5.3 \%{ }^{5,7,12}$ Patients with a large defect accompanied by hydrocephalus or Arnold-Chiari malformation are at a higher risk of CSF leakage. ${ }^{4,7,14}$

Primary closure is often insufficient to repair a large meningomyelocele. The advancement flap technique is applied in the repair of these defects to reduce tissue tension in the postoperative period. ${ }^{7}$ After meningomyelocele repair, the infant should be closely monitored for the development of hydrocephalus. In the presence of findings of raised intracranial pressure, ventriculoperitoneal shunting or an alternative method of CSF diversion should be performed. This procedure protects the brain from the effects of progressive ventricular dilation, reduces high dural sac pressure, and prevents the risk of a postoperative CSF leak from the recently repaired defect. In cases of a concomitant meningomyelocele and hydrocephalus, simultaneous ventriculoperitoneal shunting and repair of the defect are recommended. Watertight dural closure, together with the maintenance of the patient in a prone position, can reduce the risk of CSF leakage from the wound..$^{10}$

In the present case, although the patient's sodium level returned to normal after initiation of sodium-deficit therapy, the patient required an additional $20-25 \mathrm{mmol} / \mathrm{day}$ of sodium during the period because of the CSF leak. The patient was receiving full enteral feeding, and the sodium was added to the enteral solution. The patient developed hyponatremia when sufficient sodium support was not provided. Hyponatremia associated with CSF loss, in addition to an increased need for sodium, has been previously described in preterm newborns who underwent external ventricular drainage to treat posthemorrhagic hydrocephalus. ${ }^{8}$ Life-threatening dehydration and hyponatremia have been reported in children undergoing external ventricular drainage for different reasons. ${ }^{15,16}$ In addition, hyponatremia associated with CSF loss has been reported in a 3-year-old child following ventriculoperitoneal shunt placement for an abdominal CSF pseudocyst and in an 18-month-old infant with rhinorrhea. ${ }^{1,6}$

The sodium content of CSF varies between 135 and $150 \mathrm{mmol} / \mathrm{L}$. Daily CSF loss of $100 \mathrm{ml}$ causes sodium loss of $14 \mathrm{mmol}$. This amount exceeds the daily sodium intake (6 mmol) of a newborn receiving $600 \mathrm{ml}$ of breast milk (mean sodium content $0.74 \mathrm{mmol} / \mathrm{dl}$ ) or term infant formula (sodium content $0.78-0.87 \mathrm{mmol} / \mathrm{dl}$ ). Based on the present case and on previously reported cases, ${ }^{1,6,15,16} \mathrm{CSF}$ loss may cause severe hyponatremia, particularly in the newborn and early infancy periods when dietary sodium content is relatively low. The sodium content of standard maintenance fluids in this age group is about one-fourth to one-fifth of $0.9 \%$ saline, which cannot compensate for sodium loss through a CSF leak. In patients with limited oral intake, intravenous replacement therapy with $0.9 \%$ saline (to which $5 \mathrm{mmol} / \mathrm{L} \mathrm{KCl}$ may be added) should be administered independently of maintenance fluids.

While hyponatremia accompanying primary diseases causes adverse outcomes in all age groups, it is associated with growth retardation, neurodevelopmental problems, and hearing loss in newborns. ${ }^{3,11}$ Therefore, serum sodium levels in infants with CSF loss for any reason should be closely monitored, even if the individuals are receiving full enteral nutritional. The sodium needs of infants may be increased two- to threefold compared to an infant without a CSF leak, depending on the amount of CSF leakage.

\section{References}

1. Buyukyavuz BI, Duman L, Karaaslan T, Turedi A: Hyponatremic seizure due to huge abdominal cerebrospinal fluid pseudocsyt in a child with ventriculoperitoneal shunt: a case report. Turk Neurosurg 22:656-658, 2012

2. Dellabarca C, Servilla KS, Hart B, Murata GH, Tzamaloukas AH: Osmotic myelinolysis following chronic hyponatremia corrected at an overall rate consistent with current recommendations. Int Urol Nephrol 37:171-173, 2005 
3. Ertl T, Hadzsiev K, Vincze O, Pytel J, Szabo I, Sulyok E: Hyponatremia and sensorineural hearing loss in preterm infants. Biol Neonate 79:109-112, 2001

4. Gaskill SJ: Primary closure of open myelomeningocele. Neurosurg Focus 16(2):E3, 2004

5. Hashim AS, Ahmed S, Jooma R: Management of myelomeningocele. J Surg Pak (Int) 13:7-11, 2008

6. Jadhav AP, Nelson BD, Kim SS, Chiang VW: Cerebrospinal fluid rhinorrhea as a unique cause of hyponatremia. Pediatr Neurol 37:360-362, 2007

7. Lee BJ, Sohn MJ, Han SR, Choi CY, Lee DJ, Kang JH: Analysis of risk factors and management of cerebrospinal fluid morbidity in the treatment of spinal dysraphism. J Korean Neurosurg Soc 54:225-231, 2013

8. MacMahon P, Cooke RW: Hyponatraemia caused by repeated cerebrospinal fluid drainage in post haemorrhagic hydrocephalus. Arch Dis Child 58:385-386, 1983

9. Marcialis MA, Dessi A, Pintus MC, Irmesi R, Fanos V: Neonatal hyponatremia: differential diagnosis and treatment. J Matern Fetal Neonatal Med 24 (Suppl 1):75-79, 2011

10. Miller PD, Pollack IF, Pang D, Albright AL: Comparison of simultaneous versus delayed ventriculoperitoneal shunt insertion in children undergoing myelomeningocele repair. J Child Neurol 11:370-372, 1996

11. Murphy DJ, Hope PL, Johnson A: Neonatal risk factors for cerebral palsy in very preterm babies: case-control study. BMJ 314:404-408, 1997

12. Pang D: Surgical complications of open spinal dysraphism. Neurosurg Clin N Am 6:243-257, 1995

13. Rahman M, Friedman WA: Hyponatremia in neurosurgical patients: clinical guidelines development. Neurosurgery 65:925-936, 2009

14. Siasios J, Kapsalaki EZ, Fountas KN: Surgical management of patients with Chiari I malformation. Int J Pediatr 2012:640127, 2012

15. Simpson S, Yung M, Slater A: Severe dehydration and acute renal failure associated with external ventricular drainage of cerebrospinal fluid in children. Anaesth Intensive Care 34:659-663, 2006

16. Tobias JD: Cerebrospinal fluid losses through ventricular catheters leading to hyponatremia in two children. South Med J 84:279-280, 1991

\section{Disclosures}

The authors report no conflict of interest concerning the materials or methods used in this study or the findings specified in this paper.

\section{Author Contributions}

Conception and design: Gökce, Turgut. Drafting the article: Gökce. Reviewed submitted version of manuscript: Ozdemir, Onal.

\section{Correspondence}

İsmail Kürşad Gökce: Inonu University School of Medicine, Malatya, Turkey. ikgokce07@hotmail.com. 